\title{
Análise epidemiológica do COVID-19 no estado de Minas Gerais
}

\author{
Epidemiological analysis of COVID-19 in the state of Minas Gerais
}

Giulia Pacheco Souza ${ }^{1}$
Orcid: https://orcid.org/0000-0003-0739-0577

Isabelle Gualberto Souza ${ }^{3}$

Orcid: https://orcid.org/0000-0001-6522-1515

Alexandre Vitor Dias Silveira ${ }^{5}$

Orcid: https://orcid.org/0000-0002-4700-2674
Renato Alexsander Martins Lara ${ }^{2}$

Orcid: https://orcid.org/0000-0002-3516-0616

\author{
Gabriel Felipe Silveira Ferreira ${ }^{4}$
}

Orcid: https://orcid.org/0000-0002-2436-6571

Lucas de Las Casas Bessa ${ }^{6}$

Orcid: https://orcid.org/0000-0001-6714-8139

Karina Prince ${ }^{7}$

Orcid: https://orcid.org/0000-0001-8231-852X

\begin{abstract}
Resumo
Introdução: A pandemia do Coronavírus afetou diversos países ao redor do mundo em um curto espaço de tempo. Foi identificado pela primeira vez no Brasil em fevereiro de 2020 e, desde então, o número de casos e de óbitos aumentou consideravelmente. Assim, compreender o perfil populacional da doença quanto às localidades e vulnerabilidades faz-se necessário para um controle epidemiológico mais específico. Objetivo: Descrever o perfil epidemiológico do COVID-19 no estado de Minas Gerais no período de março a outubro de 2020. Métodos: Estudo quantitativo, descritivo, de base documental com procedimento comparativoestatístico. Os dados acerca dos casos e óbitos confirmados de SARS-CoV-2 no estado mineiro foram coletados a partir dos boletins epidemiológicos disponibilizados pela Secretaria de Estado de Saúde de Minas Gerais pela plataforma Coronavírus Minas Gerais. Resultados: Foram registrados 346.310 casos de COVID-19 no período, com maior prevalência no sexo feminino (51\%) e na faixa etária de 30 a 39 anos (24,3\%). Quanto aos óbitos, 8.916 foram confirmados, com maior frequência em homens $(57 \%)$ e em pessoas com mais de 60 anos $(79,52 \%)$, sendo as comorbidades mais presentes as cardiopatias $(47,77 \%)$ e o diabetes $(33,28 \%)$. Conclusões: Verificou-se prevalência de casos no sexo feminino e na faixa etária entre 30 e 39 anos, enquanto os óbitos foram mais frequentes em homens e na faixa acima de 60 anos. Ainda, foi possível visualizar a relação das políticas públicas com os números de infecções e de mortes, reforçando a necessidade de adequação e direcionamento de estratégias para o controle do COVID-19 no estado.
\end{abstract}

Palavras-chave: coronavírus; epidemiologia; pandemia.

\footnotetext{
${ }^{1}$ Centro Universitário Faculdades Integradas Pitágoras de Montes Claros (UNIFIPMoc), Minas Gerais, Brasil. E-mail: giuliapachecosouza12@gmail.com

${ }^{2}$ Centro Universitário Faculdades Integradas Pitágoras de Montes Claros, Minas Gerais, Brasil. E-mail: renato.lara@aluno.unifipmoc.edu.br

${ }^{3}$ Centro Universitário Faculdades Integradas Pitágoras de Montes Claros, Minas Gerais, Brasil. E-mail: gualbertoisabellesouza@gmail.com

${ }^{4}$ Centro Universitário Faculdades Integradas Pitágoras de Montes Claros, Minas Gerais, Brasil. E-mail: gabrielfelipe464@gmail.com

${ }^{5}$ Centro Universitário Faculdades Integradas Pitágoras de Montes Claros, Minas Gerais, Brasil. E-mail: alexvdsilveira@gmail.com

${ }^{6}$ Centro Universitário Faculdades Integradas Pitágoras de Montes Claros, Minas Gerais, Brasil. E-mail: lucas.bessa@aluno.unifipmoc.edu.br

${ }^{7}$ Centro Universitário Faculdades Integradas Pitágoras de Montes Claros, Minas Gerais, Brasil. E-mail: karina.prince@professor.uni-

fipmoc.edu.br
} 


\begin{abstract}
Introduction: The Coronavirus pandemic has affected several countries in the world in a short period. It was first identified in Brazil in February 2020 and, since then, the number of cases and deaths has increased considerably. Thus, understanding the population profile of the disease in terms of locations and vulnerabilities is necessary for specific epidemiological control. Objective: To describe the epidemiological profile of COVID-19 in the state of Minas Gerais from March to October 2020. Methods: Quantitative, descriptive, documentary study with a comparative-statistical procedure. Data about confirmed cases and deaths of SARSCoV-2 in the state were collected from epidemiological bulletins made available by the Minas Gerais State Department of Health through the Coronavirus Minas Gerais platform. Results: 346,310 cases of COVID-19 were registered, with a higher prevalence in females $(51 \%)$ and in the age group of 30 to 39 years $(24.3 \%)$. As for deaths, 8,916 were confirmed, more frequently in men $(57 \%)$ and people over 60 years old $(79.52 \%)$, with the most common comorbidities being heart diseases $(n=4,259 / 47.77 \%)$ and diabetes $(33.28 \%)$. Conclusions: Prevalence of cases in females and the age group between 30 and 39 years, while deaths were more frequent in men and the age group above 60 years. Still, it was possible to visualize the relationship between public policies and the numbers of infections and deaths, reinforcing the need to adapt and direct strategies for the control of COVID-19 in the state.
\end{abstract}

Keywords: coronavírus; epidemiology; pandemic.

\section{Introdução}

O COVID-19 é uma doença respiratória, causada pelo vírus SARS-Cov2, inicialmente identificada em Wuhan, China, em 2019 ${ }^{1}$. Sua transmissão ocorre por meio do contágio via oral/fecal, ou seja, pelo contato com gotículas de indivíduos infectados durante a fala ou espirros e com superfícies e objetos contaminados pelo vírus $^{2}$. Possui diferentes manifestações clínicas, sendo a forma assintomática responsável por aproximadamente $80 \%$ dos casos $^{1}$.

As manifestações clínicas variam de quadros mais brandos, com presença de febre, cansaço, tosse, a situações mais críticas como dificuldades respiratórias, bem como sintomas sistêmicos semelhantes à sepse, podendo evoluir ao choque e gerar uma síndrome de disfunção de múltiplos órgãos ${ }^{3}$. O agravamento da doença está relacionado diretamente com a idade e a presença de comorbidades, principalmente cardíacas, metabólicas e respiratórias ${ }^{4}$. Nesses casos, os pacientes necessitam de amparo hospitalar devido às complicações respiratórias e hemodinâmicas, em que a principal evolução é a insuficiência respiratória aguda grave ${ }^{5}$.

Desde seu surgimento, a infecção se disseminou em um curto espaço de tempo por mais de 120 países em todo o mundo, incluindo o Brasil. A alta transmissibilidade da doença, somada ao grande contingente de viagens internacionais realizadas diariamente, possibilitou a disseminação da COVID-19 em todo o globo, sendo caracterizada oficialmente, em 11 de março de 2021, como pandemia, e sendo até hoje considerada uma ameaça à saúde pública global, especialmente nos países em que os planos de contenção da transmissão da doença não têm sido exitosos ${ }^{6,7}$.

A separação oceânica do continente americano do asiático atrasou a chegada do vírus nas Américas, embora os Estados Unidos tenham notificado a presença de COVID-19 muito mais cedo que as Américas do Sul e Central, dado ao grande número de viajantes que recebem todos os dias $^{8}$. Ainda, embora os países europeus tenham sido uns dos primeiros acometidos pela doença, além de terem apresentado os primeiros surtos por COVID-19, como ocorreu na Itália e França, os Estados Unidos e o Brasil são hoje o epicentro do novo coronavírus, apresentando os maiores número de casos e óbitos registrados em todo o mundo 9 .

$\mathrm{Na}$ América Latina, o primeiro caso foi confirmado em fevereiro de 2020, quando o Ministério da Saúde (MS) do Brasil registrou o caso de um homem 
brasileiro, de 61 anos, que regressou de viagem à Itália, região onde ocorria um surto da doença na época ${ }^{10}$. Desde então, o vírus alcançou altas proporções no território nacional, sendo que, com um mês do primeiro caso, o Brasil já registrava mais de 2 mil casos e 77 óbitos por COVID-19 confirmados, além de todos os estados apresentarem casos da infecção $0^{2,10}$.

O controle epidemiológico adotado pelo país não aderiu ao método de testagem em massa que seria o padrão internacional. Até o final de agosto de 2020, mais de 24 milhões de casos de COVID-19 foram confirmados no mundo. Nesse período, o Brasil acumulava um total de 3.846.156 casos, ficando atrás apenas dos Estados Unidos com 5.917.439 registros $^{1}$. Ainda nesse período, o Brasil testou 4.110,47/100 mil habitantes, sendo que 2.218,29 testes/100 mil hab. foram aplicados pelo estado de Minas Gerais ${ }^{11}$. Hoje, o estado de Minas Gerais está entre os estados da federação que mais registram casos e óbitos da doença em $2021^{12}$.

Assim, verifica-se que o atual curso da pandemia no país atualiza de forma dinâmica os parâmetros de controle, o perfil epidemiológico, as localidades e os aspectos de vulnerabilidade ${ }^{13}$. Nesse sentido, a compreensão do perfil populacional acometido e a disponibilidade de recursos em saúde para o enfrentamento dessa doença, faz-se necessário para um controle epidemiológico mais específico ${ }^{14}$.

Dessa maneira, o presente estudo teve como objetivo principal descrever o perfil epidemiológico dos casos e óbitos conformados de COVID-19 no estado de Minas Gerais, no período de março a outubro de 2020.

\section{Metodologia}

Trata-se de um estudo retrospectivo, descritivo, quantitativo, de base documental com procedimento comparativo-estatístico. A amostra foi composta por todos os casos e óbitos confirmados de SARS-Cov-2 no estado de Minas Gerais, durante o período de março a outubro de 2020.

A coleta de dados ocorreu nos meses de outubro e novembro de 2020 por meio da utilização dos boletins epidemiológicos, disponibilizados pela Secretária de Estado de Saúde de Minas Gerais, no endereço eletrônico

(http://coronavirus.saude.mg.gov.br).

$\mathrm{O}$ estudo incluiu as seguintes variáveis: faixa etária, sexo, comorbidades, evolução dos casos, taxa de mortalidade, taxa de letalidade e local de residência. Essa última foi posteriormente classificada nas macrorregiões de saúde (Norte, Noroeste, Triângulo do Norte, Triângulo do Sul, Sul, Oeste, Centro Sul, Sudeste, Leste do Sul, Centro, Vale do Aço, Leste, Jequitinhonha e Nordeste).

Para o cálculo da taxa de mortalidade de COVID-19 no estado mineiro por 100 mil habitantes, utilizou-se o número de óbitos confirmados no estado dividido pela população total e multiplicouse por 100 mil habitantes considerando a projeção do Instituto Brasileiro de Geografia e Estatística (IBGE) para 2020. Em relação à taxa de letalidade utilizou-se o número de óbitos pela doença dividido pelo número total de casos confirmados multiplicado por 100 .

Os dados foram gerenciados e analisados por meio de estatística descritiva simples pelo software Microsoft Office Excel 2010 e pelo programa Statistical Pocckage for the Social Sciences (SPSS) para Windows, versão 25 (Chicago, IL, USA).

Conforme a Resolução 466/12 do Conselho Nacional de Saúde, o presente estudo dispensou apreciação e aprovação do Comitê de Ética em Pesquisa, por se tratar de dados secundários, de domínio e acesso público, e pelo fato de haver sigilo acerca da identificação dos pacientes envolvidos.

\section{Resultados}


No período analisado, de março a outubro de 2020, foram registrados um total de 346.310 casos de COVID-19 no estado de Minas Gerais, associados a 8.916 óbitos confirmados. O número de casos variou de 275 a 86.301, com média de 43.288,35 casos/mês. Observou-se um aumento linear do número de casos confirmados entre março e agosto (31.495\%), com decréscimo gradativo entre agosto e outubro $(32,67 \%)$. $\mathrm{O}$ mês que registrou o maior número de casos foi agosto $(n=86.885 / 25,09 \%)$ e o menor foi março $(n=275 / 0,08 \%)$.

Gráfico 1. Número de casos e óbitos por COVID-19 em Minas Gerais de acordo com o mês de notificação.

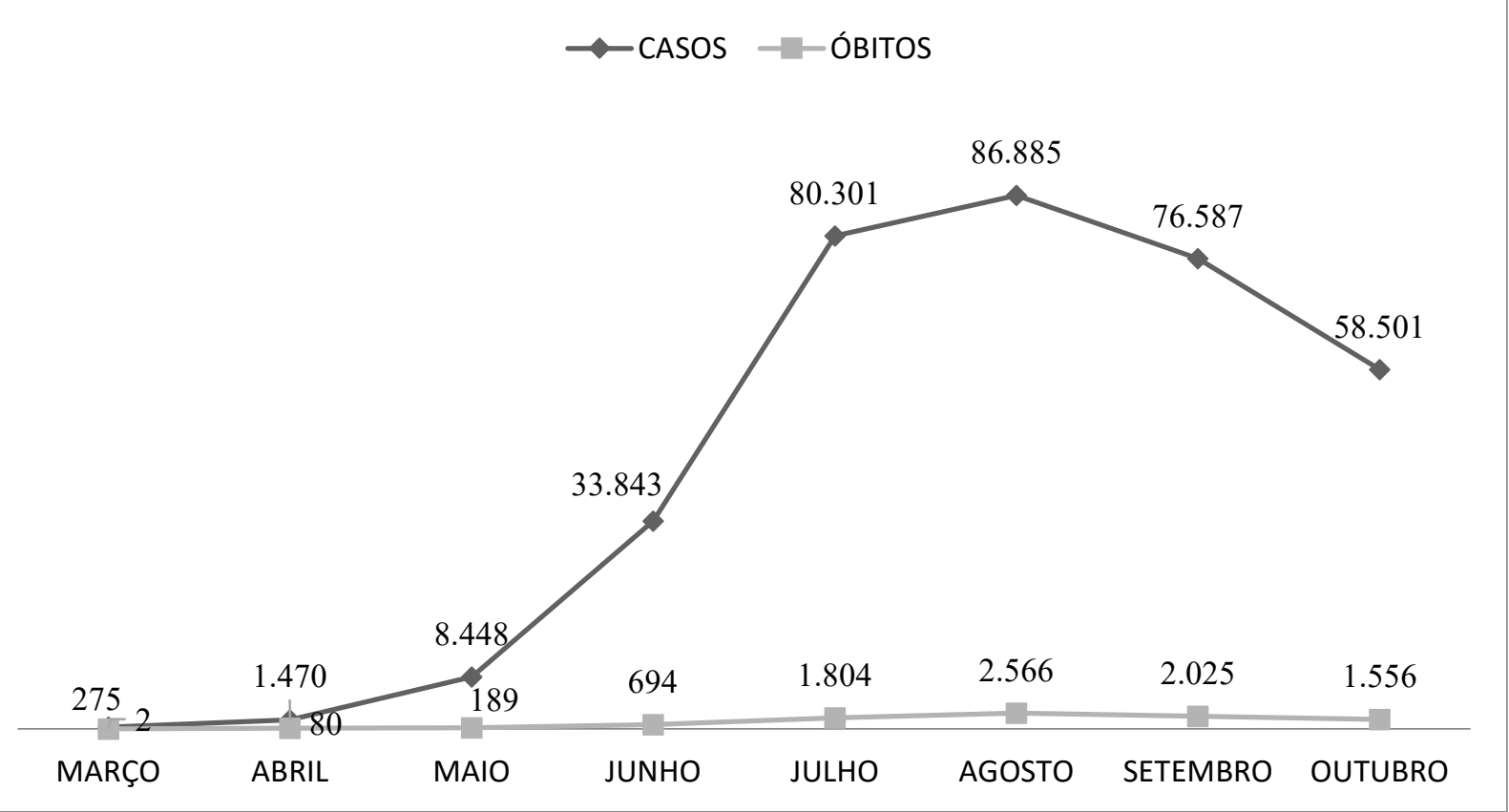

Fonte: Secretaria de Estado de Saúde de Minas Gerais, 2020.

Quanto aos óbitos, observou-se padrão similar aos casos confirmados, com o mês de março registrando o menor valor $(n=2 / 0,02 \%)$, e agosto apresentando a maior taxa $(\mathrm{n}=2.556 / 28,78 \%)$. Ainda, verificou-se que, de março a agosto, houve crescimento no número de óbitos no estado, passando de 2 óbitos confirmados em março, para um total de 2.566 vítimas em agosto. Todavia, quando analisados os totais de óbitos de agosto a outubro, observa-se que houve decréscimo relativo desses valores $(39,36 \%)$.
A tabela 1 apresenta as variáveis sociodemográficas dos casos confirmados de COVID-19 no estado. Observa-se que, analisando o número de casos de acordo com o sexo, houve relativo predomínio no sexo feminino $(\mathrm{n}=176.618 / 51 \%) . \quad \mathrm{Na}$ análise dos casos por idade, percebeu-se que a doença foi mais prevalente em indivíduos com idade entre 30 e 39 anos $(n=84.916 / 24,3 \%)$, seguido dos pacientes com idade de 40 a 49 anos $(n=68.916 / 19,9 \%) \quad$ e 20 a 29 anos $(\mathrm{n}=60.604 / 17,5 \%)$.

Tabela 1. Dados sociodemográficos e clínicos dos pacientes com COVID-19 que não evoluíram para óbitos em Minas Gerais, março a outubro de 2020.

\begin{tabular}{ccc}
\hline Variáveis & Total & \% \\
\hline Total & $\mathbf{3 4 6 . 3 1 0}$ & 100 \\
\hline Sexo & & 49,00 \\
\hline Masculino & 169.692 & 51,00 \\
\hline Feminino & 176.618 & \\
\hline Faixa etária & & \\
\hline
\end{tabular}




\begin{tabular}{ccc}
\hline 0 a 9 anos & 10.043 & 2,90 \\
10 a 19 anos & 15.930 & 4,60 \\
20 a 29 anos & 60.604 & 17,50 \\
30 a 39 anos & 84.153 & 24,30 \\
40 a 49 anos & 68.916 & 19,90 \\
50 a 59 anos & 50.215 & 14,50 \\
60 anos ou mais & 55.063 & 15,90 \\
Não Informado & 1.386 & 0,40 \\
& & 7,00 \\
\hline Comorbidades & & 9,00 \\
Sim & 24.242 & 84,00 \\
\hline Não & 31.168 & 6,38 \\
\hline Eão Informado & 290.900 & 93,62 \\
\hline Acompanhão & & 22.103 \\
Cura & 324.207 & \\
\hline
\end{tabular}

Fonte: Secretaria de Estado de Saúde de Minas Gerais, 2020.

Ainda, verificou-se que, 7\% dos pacientes apresentavam algum tipo de comorbidade. Vale ressaltar que, em $84 \%$ dos casos de COVID-19 confirmados em Minas Gerais não houve notificação quanto à presença ou não de comorbidades.

De acordo com os dados sociodemográficos dos óbitos por COVID19 no estado mineiro, nota-se que as mortes foram mais prevalentes em homens $(\mathrm{n}=5.056 / 57 \%)$ e na faixa etária de 60 anos ou mais $(\mathrm{n}=7.090 / 79,52 \%)$. A respeito das comorbidades das vítimas, verificou-se que as cardiopatias foram as mais prevalentes $(n=4.259 / 47,77 \%)$, seguidas do diabetes $(n=2.967 / 33,28 \%)$. É importar ressaltar que os óbitos podem ter mais de uma comorbidade envolvida (Tabela 2).

Tabela 2. Dados sociodemográficos e clínicos de pacientes que evoluíram a óbitos por COVID-19 em Minas Gerais, março a outubro de 2020.

\begin{tabular}{ccc}
\hline Variáveis & Total & \% \\
\hline Total & $\mathbf{8 . 9 1 6}$ & 100 \\
\hline Sexo & & 57 \\
\hline Masculino & 5.056 & 43 \\
Feminino & 3.860 & \\
\hline Faixa etária & & 0,15 \\
0 a 9 anos & & 0,12 \\
10 a 19 anos & 13 & 0,72 \\
20 a 29 anos & 11 & 2,37 \\
30 a 39 anos & 64 & 5,20 \\
40 a 49 anos & 211 & 11,92 \\
50 a 59 anos & 464 & 79,52 \\
60 anos ou mais & 1.063 & \\
\hline Comorbidades & 7.090 & \\
\hline Asma & & 3,84 \\
Cardiopatia & & 47,77 \\
Diabetes & 342 & 33,28 \\
Doença hematológica & 4.259 & 1,08 \\
Doença hepática & 2.967 & 1,58 \\
Doença neurológica & 96 & 9,01 \\
Doença renal & 141 & 9,39 \\
\hline Imodeficiência & 803 & 5,83
\end{tabular}




\begin{tabular}{ccc}
\hline Variáveis & Total & \% \\
\hline Obesidade & 766 & 8,59 \\
Pneumopatia & 855 & 9,59 \\
Síndrome de Down & 36 & 0,40 \\
\hline
\end{tabular}

Fonte: Secretaria de Estado de Saúde de Minas Gerais, 2020.

Em relação à distribuição dos casos confirmados por COVID-19 de acordo com as macrorregiões de saúde de Minas Gerais, verifica-se um maior número de casos na região Centro $(n=106.371 / 30,92 \%) \quad$ e Triângulo do Norte $(\mathrm{n}=50.393 / 14,65 \%)$.
Em relação aos óbitos por macrorregião, verificou-se que o Centro ( $n=3.095 / 35,02 \%)$ e o Triângulo do Norte $(\mathrm{n}=1.002 / 11,34 \%)$ também registraram os maiores valores.

Gráfico 2. Número de casos e óbitos por COVID-19 em Minas Gerais de acordo com as macrorregiões de saúde de Minas Gerais, março a outubro de 2020.

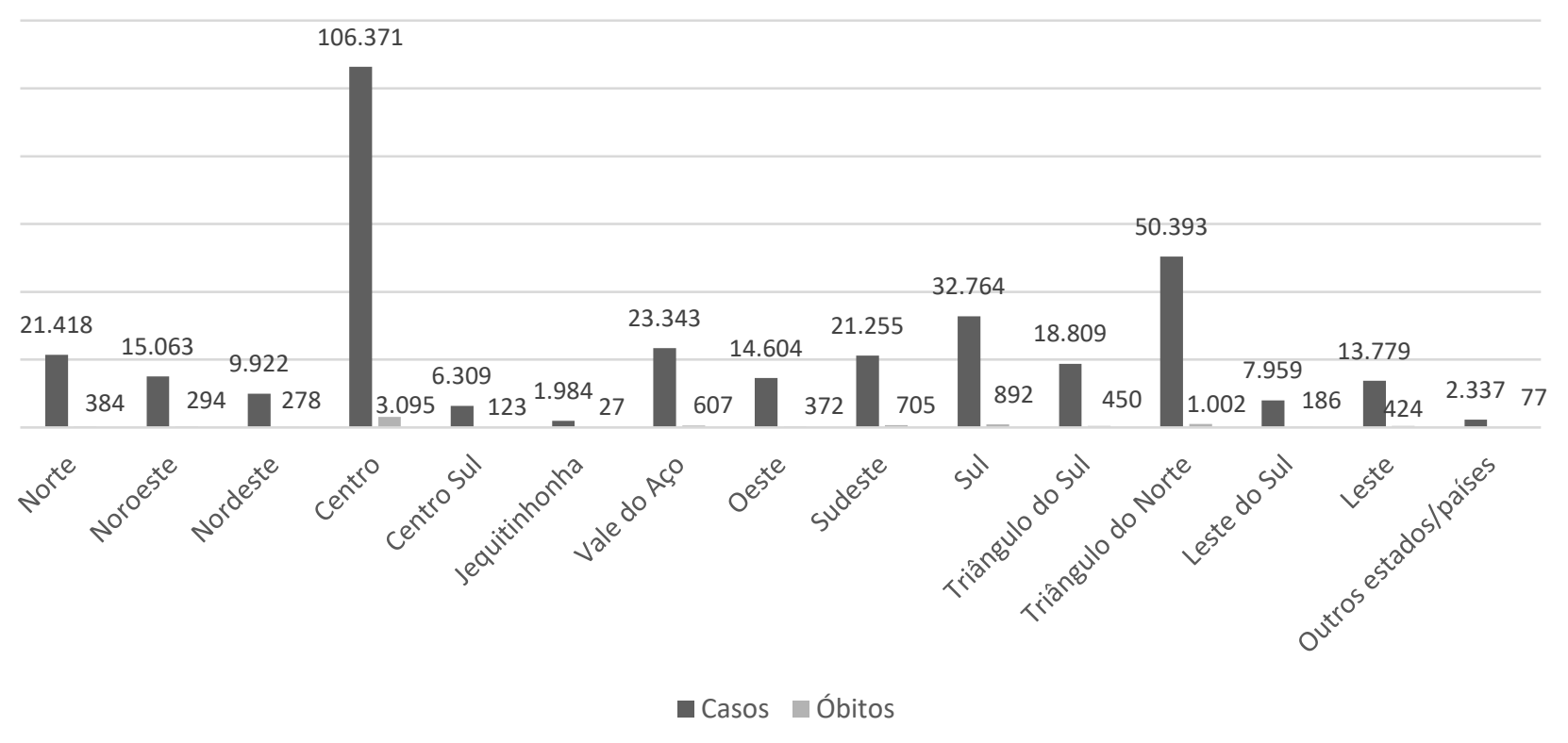

Fonte: Secretaria de Estado de Saúde de Minas Gerais, 2020.

No que diz respeito à taxa de mortalidade geral da doença no período analisado, foi de 41,87/100 mil habitantes (hab.). A taxa de mortalidade foi maior em homens do que em mulheres, sendo que, no sexo masculino, a taxa foi de 23,74/100 mil hab., e no sexo feminino, de 18,13/100 mil hab. Em relação à taxa de letalidade geral do COVID-19 nesse período, foi de 2,57\%. No que se refere à taxa de letalidade quanto aos sexos, verificou-se, também, predominância no sexo masculino, com a taxa de letalidade em homens variando de $2,98 \%$, e nas mulheres variando de $2,18 \%$.

\section{Discussão}

A variação referente ao número de casos de COVID-19 no estado de Minas Gerais acompanhou as decisões governamentais. $\mathrm{O}$ mês de março, foi $\mathrm{o}$ período com o menor número de casos, uma vez que as medidas de contenção quanto à circulação de pessoas e o fluxo com outras cidades do Brasil foi interrompido pelo fechamento de bares, lojas e estabelecimentos não essenciais ${ }^{10}$.

$\mathrm{O}$ crescimento linear representado no Gráfico 1, entre o mês de abril e agosto, ocorre simultaneamente ao período de flexibilização das ações para isolamento social concomitante ao aumento dos 
registros dos casos nas cidades do interior do estado. $\mathrm{O}$ decréscimo entre o mês de agosto e outubro ocorre devido ao maior conhecimento científico acerca da doença e das medidas sociais adotadas para controle $^{15}$.

Analisando a variável sexo, os dados acerca do número de casos estão em concordância com a literatura, que apresenta maior prevalência no gênero feminino. Todavia, esse perfil epidemiológico não é corroborado por estudos realizados na China, Itália, Espanha, Reino Unido e Estados Unidos, em que observou um perfil masculino para os casos confirmados da doença ${ }^{16}$. Em relação à faixa etária houve semelhança com a literatura prévia, na qual foi percebido maior acometimento entre indivíduos de 30 a $39 \operatorname{anos}^{10,16}$.

Durante a análise do impacto das comorbidades no curso do COVID-19, foi identificado em um estudo epidemiológico que há influência diretamente proporcional ao risco e a evolução da doença ${ }^{4}$. Entretanto, essa informação não pode ser ratificada pelo presente estudo, visto que a maior parte dos dados relativos à presença ou não de comorbidade não foi informado. Todavia, foi observado que a prevalência das comorbidades apresentou o mesmo padrão da China e Itália de uma maior prevalência em cardiopatias e de pacientes diabéticos ${ }^{17}$.

Por sua vez, ao comparar os dados referentes aos óbitos nota-se consonância com o estudo realizado no Espírito Santo, na qual a média da idade dos falecimentos foi de 66 anos, bem como o fato de homens representarem uma maior parcela de internações em UTIs ${ }^{4}$. Ademais, essa média de idade pode ser influenciada por fatores socioeconômicos, sendo a disparidade econômica, os aspectos de vulnerabilidade social e o grau de alfabetização relevantes quando considerados fatores de inclusão e exclusão à taxa de mortalidade, que em alguns casos superam 30 mortes por mil habitantes ${ }^{18}$.
Com relação à letalidade e à mortalidade descrita no estado de março a agosto houve discrepância com a literatura pré-existente, em que o presente estudo apresentou valores superiores nessas variáveis ${ }^{11}$. Apesar disso, considerando as diferentes realidades de cada macrorregião de saúde mineira e as disparidades sociais existentes, os óbitos por COVID-19 em Minas se mantiveram abaixo de outros estados brasileiros.

$O$ perfil de disseminação do COVID-19 foi influenciado pela globalização dos principais polos econômicos mundiais e pelo fluxo de pessoas nessas regiões. Durante essa análise foi percebido que o nível de industrialização foi um fator semelhante entre as nações para a propagação da doença nos centros urbanos e esse mesmo aspecto foi observado na Itália, Reino Unido, Espanha e Estados Unidos ${ }^{19}$.

$O$ estudo evidenciou que a distribuição do COVID-19 em Minas Gerais segue o padrão de transmissão do país ${ }^{10}$. Primeiramente, houve o crescimento nos polos de maior circulação de pessoas e fluxo econômico, que inclui as maiores cidades como a capital Belo Horizonte e as cidades de referência do Sul, Triângulo, Centro e Zona da Mata. A maior representatividade da região Central, correspondente a 30,92\%, ocorre pela localização da capital mineira e sua influência como região econômica e de capitalização de doentes sendo a maior referência do estado.

As demais macrorregiões de Minas contribuíram para o aumento de hospitalizações, óbitos e pela sobrecarga do sistema de saúde. Entretanto, essa realidade não confere aos dados epidemiológicos a real dimensão do impacto do COVID-19 no estado $^{5}$. Mesmo ao considerar as diferentes realidades de cada macrorregião de saúde mineira e as disparidades sociais existentes semelhantes a outros estados, o número de óbitos se manteve abaixo de outras regiões brasileiras. Dessa forma, a existência de 
subnotificações era esperada considerando a velocidade do número de casos registrados e o que foi relatado em outros países.

A presença de subnotificações foi percebida pelo mundo, principalmente, nos primeiros meses da pandemia. Entretanto, dados epidemiológicos demonstrados na Coreia do Sul, Alemanha, Itália, Suécia e nos EUA identificaram que os países que realizaram mais testes e rastreio de contatos em grande escala, possuíam menor estimativa percentual de subnotificações ao decorrer da pandemia, bem como essa porcentagem se manifestou em caráter decrescente nas nações que aplicaram essa metodologia de contenção viral ${ }^{20}$.

No Brasil, por sua vez, foi identificado a existência de subnotificação dos casos, principalmente em regiões mais periféricas, representando baixo índice de casos confirmados em comparação aos demais estados ${ }^{21}$. Em Minas Gerais as subnotificações também foram observadas no que diz respeito à letalidade e à mortalidade descrita de março a agosto, em que o presente estudo apresentou valores superiores nessas variáveis, sendo discrepante com a literatura pré-existente ${ }^{11}$. Contudo, o aumento exacerbado do número de hospitalizações registradas no estado mineiro por insuficiência respiratória aguda grave, principal evolução das formas graves do COVID-19, contrapõe essa realidade.

\section{Conclusão}

O perfil epidemiológico dos casos confirmados de COVID-19 no estado de Minas Gerais é caracterizado por maior prevalência no sexo feminino e por pessoas na faixa etária economicamente ativa, entre os 30 e 39 anos. Dentre os óbitos esse perfil se altera, sendo maior a prevalência em pessoas acima dos 60 anos, do sexo masculino e portadores de alguma comorbidade.

A pandemia do novo Coronavírus em Minas demonstrou, durante o período analisado, a influência de medidas de contenção e sua flexibilização, e como essas ações impactaram nos números do estado. Quando em comparação a outros estados brasileiros, Minas Gerais apresentou reduzida taxa de contaminação, entretanto, a dificuldade em testagem e rastreio de casos, tornou incongruentes os números de casos informados no boletim, quando confrontados pelos casos de insuficiência respiratória aguda grave.

Apesar dos esforços, os dados apresentados por este estudo refletem a necessidade de continuação das medidas de controle no estado. O comportamento epidemiológico da doença é influenciado pelo fluxo econômico e geográfico de pessoas, e por essas diferenças, se manifesta com características distintas nos grandes centros e nas periferias. Faz-se necessário, portanto, a realização de novos estudos epidemiológicos a fim de direcionar e adequar estratégias para o controle do SARS-CoV-2, bem como para possibilitar o planejamento de ações e cuidados no combate à doença em Minas Gerais.

\section{Referências}

1. BRASIL. Ministério da Saúde. Boletim COE Covid-19. Brasília - DF, 2020.

2. Ferreira Netto RG, Corrêa JWN. Epidemiologia do surto de doença por Coronavírus (COVID-19). Revista Desafios. Palmas, v. 7, p. 18-25, 2020. Suplemento COVID-19.

3. Neerukonda SN, Katneni UA Review on SARS-CoV-2 Virology, Pathophysiology, Animal Models, and Anti-Viral Interventions. Pathogens. V. 9, n. 426, p. 1-22, 2020. 
4. Maciel EL, Jabor P, Gonçalves Júnior E, Tristão-Sá R, Lima RCD, Reis-Santos B, Lira P, Bussinguer ECA, Zandonade E. Fatores associados ao óbito hospitalar por COVID-19 no Espírito Santo, 2020. Epidemiologia e Serviços de Saúde. Brasília, v. 29, n. 4, p. 1-11, 2020.

5. Magalhães SCM, Santos FO, Lima SC, Fonseca ES. Situação Epidemiológica da Transmissão da COVID-19 no Norte de Minas Gerais, Brasil. Revista Brasileira de Geografia Médica e da Saúde. Uberlândia, p.80-87, jun de 2020. Edição Especial.

6. Hu B, Guo H, Zhou P, Shi ZL. Characteristics of SARS-CoV-2 and COVID-19. Nature Reviews. V. 19, p. 141-154, 2021.

7. Lazarus JV, Ratzan SC, Palayew A, Gostin LO, Larson HJ, Rabin K, Kimball S, ElMohandes A. A global survey of potential acceptance of a COVID-19 vaccine. Natura Public Health Emergency Collection. V. 27, n. 2, p. 1-4, 2021.

8. ACHA. American College Health Association. Guidelines - preparing for COVID-19. Silver Spring: ACHA Guidelines, 2020.

9. Gomes GGC, Bisco NCB, Paulo MF, Fabrin SCV, Fioco EM, Verri ED, Regalo SCH. Perfil epidemiológico da Nova Doença Infecciosa Coronavírus - COVID-19 (Sars-Cov-2) no mundo: Estudo descritivo, janeiro-junho de 2020. Brazilian Journal of Health Review. Curitiba, v. 3, n. 4, p. 7993-8007, 2020.

10. Lima SC, Fonseca ES, Santos FO. Situação epidemiológica e difusão da COVID-19 pela rede urbana em Minas Gerais, Brasil. Revista Brasileira de Geografia Médica e da Saúde. Uberlândia, p.243-250, Jun./2020. Edição Especial.

11. Rosa MFP, Silva WNT, Carvalho WRG, Oliveira SV. Epidemiologia da COVID-19 em Uberlândia (MG): análise preliminar do impacto do grau de abertura comercial em diferentes momentos da pandemia. Journal Health NPEPS. Uberlândia, v.5, n.2, p.20-41, dez, 2020 .

12. BRASIL. Ministério da Saúde. Boletim Epidemiológico Especial. Brasília-DF, 2021.

13. Neves AGM, Guerrero G. Predicting the evolution of the COVID-19 epidemic with the ASIR model: Lombardy, Italy and São Paulo state, Brazil. Physica D. Utrecht, v. 413, p.1$12,2020$.

14. Mendonça FD, Rocha SS, Pinheiro DLP, Oliveira SV. Região Norte do Brasil e a pandemia de COVID-19: análise socioeconômica e epidemiológica. Journal Health NPEPS. Cuiabá, v. 5, n. 1, p. 20-37, jan-jun, 2020.

15. Szwarcwald CL, Souza Júnior PRB, Malta DC, Barros MBA, Magalhães MAFM, Xavier DR, Saldanha RF, Damacena GN, Azevedo LO, Lima MG, Romero D, Machado IE, Gomes CS, Werneck AO, Silva DRP, Gracie R, Pina MF. Adesão às medidas de restrição de contato físico e disseminação da COVID-19 no Brasil. Epidemiologia e Serviço de Saúde. Brasília, v. 29, n.5, p. 1-11, 2020.

16. Escosteguy CC, Eleuterio TA, Pereira AGL, Marques MRVE, Brandão AD, Batista JPM. COVID-19: estudo seccional de casos suspeitos internados em um hospital federal do Rio 
de Janeiro e fatores associados ao óbito hospitalar. Epidemiologia e Serviço de Saúde. Brasília, p. 1-24, 2020.

17. Duarte MMS, Haslett MIC, Freitas LJA, Gomes NTN, Silva DCC, Percio J, Wada MY, Fantinato FFST, Almeida WAF, Silva DA, Gava C, França GVA, Macário EM, Baêta KF, Malta JMAS, Alves AJS. Descrição dos casos hospitalizados pela COVID-19 em profissionais de saúde nas primeiras nove semanas da pandemia, Brasil, 2020. Epidemiologia e Serviço de Saúde. Brasília, vol. 29, n.5, p.1-15, 2020.

18. Lins-Filho PC, Araújo MMS, Macêdo TS, Melo MCF, Ferreira AKA, Silva ELMS, Freitas JLM, Caldas Júnior AF. The impact of socioeconomic vulnerability on COVID-19 outcomes and social distancing in Brazil. SciELO Preprints.

19. Lima NT, Buss PM, Paes-Sousa R. A pandemia de COVID-19: uma crise sanitária e humanitária. Cadernos de Saúde Pública. Rio de Janeiro, vol. 36, n. 7, p.1-4, 2020.

20. Carvalho EA, Carvalho RA. COVID-19: Time-Dependent Effective Reproduction Number and Sub-notification Effect Estimation Modeling. MedRxiv Preprints. 2020.

21. Corrêa PRL, Ishitani LH, Abreu DMX, Teixeira RA, Marinho F, França EB. A importância da vigilância de casos e óbitos e a epidemia da COVID-19 em Belo Horizonte, 2020. Revista Brasileira de Epidemiologia. Rio de Janeiro, v.23, p. 1-12, 2020.

\section{Como citar este artigo:}

Souza GP, Lara RAM, Souza IG, Ferreira GFS, Silveira AVS, Bessa LLC, Prince K. Análise epidemiológica do COVID-19 no estado de Minas Gerais . Rev. Aten. Saúde. 2021; 19(68): 237-246. 